\title{
Modifications of hyaluronan influence the interaction with human bone morphogenetic protein-4 (hBMP-4)
}

\begin{tabular}{|r|l|}
\hline Journal: & Biomacromolecules \\
\hline Manuscript ID: & bm-2009-008827.R2 \\
\hline Manuscript Type: & Article \\
\hline Aute Submitted by the & 20 -Oct-2009 \\
\hline Complete List of Authors: & $\begin{array}{l}\text { Hintze, Vera; Technische Universität Dresden, Institute of Material } \\
\text { Science, Max Bergmann Center of Biomaterials } \\
\text { Möller, Stephanie; INNOVENT e. V., Biomaterials } \\
\text { Schnabelrauch, Matthias; INNOVENT e. V., Biomaterials } \\
\text { Bierbaum, Susanne; Technische Universität Dresden, Institute of } \\
\text { Material Science, Max Bergmann Center of Biomaterials } \\
\text { Viola, Manuela; Università dell'Insubria, Experimental and Clinical } \\
\text { Biomedical Sciences } \\
\text { Worch, Hartmut; Technische Universität Dresden, Institute of } \\
\text { Material Science, Max Bergmann Center of Biomaterials } \\
\text { Scharnweber, Dieter; Technische Universität Dresden, Institute of } \\
\text { Material Science, Max Bergmann Center of Biomaterials }\end{array}$ \\
\hline
\end{tabular}

\section{(5) ScholaronE" \\ Manuscript Central}




\title{
Modifications of hyaluronan influence the interaction
}

\section{with human bone morphogenetic protein-4 (hBMP-4)}

\author{
Vera Hintze $^{1}$, Stephanie Moeller ${ }^{2}$, Matthias Schnabelrauch ${ }^{2}$, Susanne Bierbaum ${ }^{1}$, Manuela Viola $^{3}$, \\ Hartmut Worch ${ }^{1}$ and Dieter Scharnweber ${ }^{1}$
}

${ }^{1}$ Institute of Material Science, Max Bergmann Center of Biomaterials, Technische Universität Dresden, 01069 Dresden, Germany, ${ }^{2}$ Biomaterials Department, INNOVENT e.V., 07745 Jena, Germany, ${ }^{3}$ Department of Experimental and Clinical Biomedical Sciences, Università dell'Insubria, 21100 Varese, Italy.

RECEIVED DATE (to be automatically inserted after your manuscript is accepted)

To whom correspondence should be addressed. E-mail: Vera.Hintze@tu-dresden.de

\begin{abstract}
In this study we have demonstrated that the modification of hyaluronan (hyaluronic acid; Hya) with sulfate groups led to different binding affinities for recombinant human bone morphogenetic protein- 4 (rhBMP-4). The high-sulfated sHya2.8 (average degree of sulfation (D.S.) 2.8) exhibited the tightest interaction with rhBMP-4 followed by the low-sulfated sHya1.0 as determined with surface plasmon resonance (SPR), ELISA as well as competition ELISA. Unmodified Hya, chondroitin-sulfate (CS) and heparan sulfate (HS) showed significantly less binding affinity. SPR data could be fitted to an A + B = AB Langmuir model and binding constants were evaluated ranging from 13 pM to $5.45 \mu \mathrm{M}$. The interaction characteristics of the differentially sulfated Hyas are promising for the incorporation of these modified polysaccharides in bioengineered coatings of biomaterials for medical applications.
\end{abstract}


Keywords

Biosensor, surface plasmon resonance (SPR), bone morphogenetic protein (BMP), chondroitin sulfate (CS), ELISA, hyaluronic acid/hyaluronan.

\section{Introduction}

Hyaluronan (Hya) is unique among glycosaminoglycans with respect to not being covalently bound to a polypeptide. The linear polymer has a relatively simple structure of repeating non-sulfated disaccharide units composed of D-glucuronic acid [ $\beta-1-3]$ and N-acetyl-D-glucosamine [ $\beta-1-4]$. The polysaccharide is apparently of vital importance because no inherited disease is known in which it is lacking $^{1-3}$. Hya directly affects tissue organization by activating signaling pathways via CD44 cellsurface receptors ${ }^{2}$. In vitro, Hya promotes cell migration, cell adhesion ${ }^{4-6}$ and differentiation in development. Furthermore it modulates the inflammatory response ${ }^{7}$, ECM (extracellular matrix) remodelling ${ }^{8}$ and wound healing $2,3,9,10$. Huang and co-workers have presented molecular-weightdependent positive effects of Hya on osteoblast proliferation and differentiation ${ }^{11}$. Hya is especially appropriate for tissue engineering applications because of its non-immunological properties, widespread availability and ease of chain manipulation ${ }^{6}$. Extensive efforts have been made by various laboratories to produce derivatives of Hya with additional unique properties for specific biomedical applications. A wide variety of Hya derivatives are currently used for eye surgery, joint viscoelastic supplementation, and anti-adhesion films ${ }^{12}$.

By chemically cross-linking high-molecular-weight Hya biocompatible and biodegradable hydrogels have been formulated for the delivery of bone morphogenetic proteins (BMPs) ${ }^{1,13,14}$. Hydrogel-rhBMP2 composite implants have been shown to induce endochondral bone formation at heterotopic sites $^{1}$ and heal critically sized skull defects in rabbits ${ }^{13,15}$.

Another interesting form of modification is the introduction of sulfate groups. Sulfated Hya (sHya) exhibits high activity and biocompatibility with cells: Yamada et al. have demonstrated the effect of 
sHya on normal human astrocytes activity and supporting role in neuron survival ${ }^{16}$. The sulfate group of sHya seems to enhance expression of cell adhesion proteins in rat calvarial osteoblast cells resulting in aggregate formation and further remarkable induction of the alkaline phosphatase activity ${ }^{17}$.

Furthermore, naturally occurring sulfate groups in glycosaminoglycan (GAG) chains like heparin/heparan sulfate (HS) have been identified as important determinants of protein-binding sites. They are introduced during the process of polymer modification. Heparin shows a high proportion of Osulfated disaccharide units and a relatively homogeneous overall sulfation pattern, whereas HS is composed of alternating sulfated and non-sulfated sequences of variable length ${ }^{18-20}$. Interactions between GAGs and proteins may be highly specific, as illustrated by binding of heparin/HS to antithrombin $^{21}$. Other proteins, such as histones, bind GAGs due to their overall basic character, without any apparent need for a specific saccharide sequence ${ }^{22,23}$. HS chains are known to interact with a variety of proteins such as heparin-binding growth and differentiation factors (HBGFs), morphogens, extracellular matrix components, protease inhibitors, proteases, lipoprotein lipases, and various pathogens. The interaction of growth factors/GAGs could be of importance in i) stabilizing the protein with respect to denaturation as well as proteolysis at normal physiological ionic strength, $\mathrm{pH}$, and temperature, ii) preventing the diffusion of the growth factor within a tissue compartment or between tissue compartments, as well as allowing locally stored growth factors to act on a restricted number of cells, iii) enabling or promoting growth-stimulatory activities by stabilizing interactions between growth factors and receptors and iv) playing a critical role in regulating growth factor availability by controlling the deposition of latent growth factor binding proteins into the $\mathrm{ECM}^{24-27}$.

Heparin dose dependently enhances the osteoblast differentiation induced not only by homodimers of BMP-2 or BMP-4 but also heterodimers of BMP-2/6 or BMP- $2 / 7^{28}$. Heparin also appears to potentiate BMP-2 activity by inducing the synthesis of proteoglycans in embryonic limb bud cells ${ }^{29}$.

HS and dextran sulfate also enhanced the BMP-2 activity, while chemically desulfated heparinderivatives have lost this stimulatory capacity. This suggests that sulfated polysaccharides enhance the 
biological activity of BMPs by presenting the ligands to their signaling receptors expressed on cell membranes ${ }^{28}$.

Heparin-binding determinants are located in the N-terminal segments of BMPs as indicated by the complete loss of high affinity binding in a BMP-2 variant in which the N-terminal residues of BMP-2 had been substituted by a dummy sequence of equal length. The most prominent difference between the N-terminal sequence of the variant and that of BMP-2 is the lack of five basic side chains (K3, H4, K5, R7, K11). It can be concluded that these residues contribute to heparin binding. Similar basic residues albeit with a slightly different spacing exist in the N-termini of BMP-4 and the Drosophila dpp $\operatorname{protein}^{29}$. Therefore it seems to be a feasible approach to investigate Hya derivatives with varying type and content of anionic groups for their growth factor binding activities creating new forms of Hya for biomedical applications. These forms could then enhance the osteoinductive properties of biocompatible Hya by specifically interacting with stimulating factors of growth and differentiation. Members of the TGF- $\beta 1$ growth factor superfamily, in particular TGF- $\beta 1$, BMP-2 and -4 are potent bone inducing factors and good candidates for this kind of interaction.

In this study we investigated the interaction of sHya with high and with low degree of sulfation (D.S.) in comparison with CS and HS (Figure 1) to the bone inducing growth factor recombinant human BMP4 using immunological and biophysical methods, i.e. enzyme linked immunosorbent assay (ELISA) and surface plasmon resonance (SPR).

\section{Materials and Methods}

Materials. Hya (from Streptococcus, $\mathrm{M}_{\mathrm{W}}=1.1 \times 10 \mathrm{E}+6 \mathrm{~g} \mathrm{~mol}^{-1}$ ) was obtained from Aqua Biochem, Dessau, Germany, sulfur trioxide/dimethylformamide complex $\left(\mathrm{SO}_{3}-\mathrm{DMF}\right.$, purum, $\geq 97 \%$, active $\mathrm{SO}_{3}$ $\geq 48 \%$ ) and sulfur trioxide/pyridine complex $\left(\mathrm{SO}_{3}\right.$-pyridine, pract.; $\left.\geq 45 \% \mathrm{SO}_{3}\right)$ from Fluka Chemie, Buchs, Switzerland. Bovine tracheal chondroitin-sulfate sodium salt (CS; a mixture of $70 \%$ chondroitin4-sulfate or CS-A and 30\% chondroitin-6-sulfate or CS-C) and heparan sulfate sodium salt from bovine 
kidney (HS; according to the supplier with a sulfur content of 5-7 \%) were purchased from SigmaAldrich, Schnelldorf, Germany. Recombinant human rhBMP-4 as well as mouse anti-human BMP-4 antibodies was obtained from R\&D Systems, Wiesbaden-Nordenstadt, Germany. The sensor chip CM5, the amine coupling kit and HBS-EP buffer were all purchased from GE Healthcare Europe GmbH, Freiburg, Germany.

Methyl-/vinyletheranhydrid-copolymer and adipinic acid dihydrazide were purchased from Fisher Scientific GmbH, Nidderau, Germany. Sodium cyanoborohydride, bovine serum albumin (BSA), Tween 20, Sucrose, 4-nitrophenylphosphate disodium salt hexahydrate and 3,3',5,5'-tetramethylbenzidine liquid substrate were available from Sigma-Aldrich, Schnelldorf, Germany. The biotin-conjugated goat anti-mouse antibody was purchased from Dianova, Hamburg, Germany.

\section{Preparation of Hya sulfates (sHya).}

\section{General procedures}

The tetrabutylammonium salt of Hya (TeBA-Hya) was used as reactant for the sulfation and prepared as follows: $2.0 \mathrm{~g}(4.98 \mathrm{mmol})$ of Hya (sodium salt) were dissolved in bidistilled water $(400 \mathrm{ml})$ at room temperature over night. The solution was stirred with $20 \mathrm{~g}$ of Dowex WX 8 ion exchanger (tetrabutylammonium-form) over night and after filtration, the polymer solution was lyophylized and dried under vacuum at $40^{\circ} \mathrm{C}$. Yield: $90 \%$.

High-sulfated HA (sHya2.8)

$2.0 \mathrm{~g}$ (3.22 mmol) of TeBA-Hya was suspended under argon at room temperature in DMF (400 mL). $9.9 \mathrm{~g}(64.4 \mathrm{mmol})$ of the $\mathrm{SO}_{3}$-DMF complex dissolved in $40 \mathrm{ml} \mathrm{DMF}$, were added (polymer : $\mathrm{SO}_{3}$ ratio $=1: 20$ ) and the reaction solution was stirred for $1 \mathrm{~h}$ at room temperature. Yield: $70 \%$ (related to Hya$\mathrm{Na})$.

Low-sulfated HA (sHyal.0)

$3.6 \mathrm{~g}$ (22.54 mmol) of the $\mathrm{SO}_{3}$-pyridine complex, dissolved in $40 \mathrm{ml} \mathrm{DMF}$, was added to a suspension of $2.0 \mathrm{~g}$ (3.22 mmol) of TeBA-Hya in DMF (400 mL; molar polymer : $\mathrm{SO}_{3}$ ratio = $\left.1: 7\right)$ under argon at 
room temperature. The reaction solution was stirred for 20 min. Yield: $65 \%$ (related to Hya-Na). In both cases the sulfated products were isolated from the reaction mixture by precipitation into acetone $(2.8 \mathrm{~L})$ and neutralized using ethanolic $\mathrm{NaOH}$ solution. The formed sodium salt of the sHya were washed several times with acetone and purified by dialysis against distilled water followed by lyophilization of the aqueous solutions and drying of the resulting polymers under vacuum.

Characterization of sHya and CS. NMR spectra were recorded in $\mathrm{D}_{2} \mathrm{O}$ (99.9\%, Sigma-Aldrich, Schnelldorf, Germany), with a Bruker Advance $400 \mathrm{MHz}$ spectrometer at a temperature of $373 \mathrm{~K}$. $\mathrm{D}_{2} \mathrm{O}$ at $4.75 \mathrm{ppm}$ was used as reference line. The $\mathrm{KBr}$ technique was employed for recording FT-IR spectra with a FT-IR-Spektrometer FTS 175 (Bio-Rad, Krefeld, Germany). Molecular weight determination was performed by gel permeation chromatography (GPC) analysis using the following equipment: Jasco PU 980 pump, Postnova Analytics PN 3000 (15) laser light scattering (LLS) detector, Jasco RID-1531 refraction (RI) detector, and a combination of 3 Suprema-Gel columns with dimensions of $8 \mathrm{~mm} \times 300$ mm (diameter x length) and the specifications $10 \mu \mathrm{m}-100 \AA, 10 \mu \mathrm{m}-1,000 \AA$ and $20 \mu \mathrm{m}-30,000 \AA$ referring to grain size and pore size, respectively. The eluent was PBS buffer and the flow rate for all columns was $0.8 \mathrm{ml} / \mathrm{min}$. Concerning refraction index detection, the system was calibrated with standard pullulan (PSS, Mainz, Germany). LLS detection of analytes was used for the determination of molecular weight values (number-average and weight-average molecular weights $M_{n}$ and $M_{w}$ ) and RI detection for the estimation of the polydispersity (PD) indices. The D.S. of the sHya was determined by estimation of the sulfur content using an automatic elemental analyzer (CHNS-932, Leco, Mönchengladbach, Germany). Analytical data are given in Table 1 and Figure 2.

Immobilization of rhBMP-4 on $\mathrm{CM5}^{\mathrm{TM}}$ biosensor chip surfaces. For interaction analysis of growth factor and GAGs a BIAcore $3000^{T M}$ instrument with BIAcore Control and BIAevaluation software 4.1 was used. RhBMP-4 was immobilized on the surface of a CM5 sensor chip using the amine coupling reaction as described by the manufacturer, where the protein's primary amino groups reacted with carboxymethylated dextran, yielding a stable covalent bond. Carboxymethylated dextran covers the 
surface of the gold film in the sensor chip, providing a hydrophilic environment conducive for the interaction of biological molecules. Briefly, the carboxymethylated dextran surface was activated using an injection pulse (7 $\mathrm{min}, 35 \mu \mathrm{L})$ containing a mixture of 1-ethyl-3-(3-dimethylaminopropyl) carbodiimide hydrochloride (EDC) and N-hydroxysuccinimide (NHS). Protein immobilization was accomplished by injecting $30 \mu \mathrm{L}$ of rhBMP-4 with a concentration of $10 \mu \mathrm{g} / \mathrm{mL}$ in $10 \mathrm{mM}$ sodium acetate buffer at $\mathrm{pH}$ 5.5. The remaining non-reacted sites on the sensor surface were blocked with a 35 $\mu \mathrm{L}$ injection of $1 \mathrm{M}$ ethanolamine- $\mathrm{HCl} \mathrm{pH}$ 8.5. One flow cell was used as a reference surface and was directly deactivated after the injection pulse of EDC/NHS by injecting $1 \mathrm{M}$ ethanolamine- $\mathrm{HCl}$ without the immobilization of rhBMP-4. All steps were carried out in a continuous flow of HBS-EP (0.01 M Hepes (pH 7.4), 0.15 M NaCl, 3 mM EDTA, $0.005 \%$ surfactant P20) running buffer at $5 \mu \mathrm{L} / \mathrm{min}$ and all buffers were degassed prior to use.

SPR measurements of growth factor interaction with GAGs. The baseline was allowed to stabilize for at least $2 \mathrm{~h}$ in HBS-EP running buffer before injecting test samples. Each GAG sample was prepared in HBS-EP buffer and injected for $3 \mathrm{~min}$ at $30 \mu \mathrm{L} / \mathrm{min}$ followed by a $2 \mathrm{~min}$ dissociation phase. The sensor surface was regenerated using $30 \mu \mathrm{L} 3 \mathrm{M} \mathrm{NaCl}$. Re-equilibration between the sensor surface and running buffer was established prior to injection of the next sample by a 2 min pre-run phase. The response was monitored as a function of time (sensorgram) at $25^{\circ} \mathrm{C}$ and subtracted from the response of the reference surface. Kinetic parameters were evaluated using the BIAevaluation software 4.1.

Covalent coupling of GAGs to MaxiSorp ${ }^{\mathrm{TM}}$ 96-well ELISA-plates. The polysaccharides were immobilized in each well of MaxiSorp ${ }^{\text {TM }}$ 96-well microtiter plates from Thermo Fisher Scientific (Nunc GmbH \& Co. KG, Lengenselbold, Germany) via their reducing ends. In brief, $50 \mu \mathrm{g}$ GAGs were solubilized in $25 \mathrm{mM}$ citric acid supplemented phosphate buffer $\mathrm{pH} 5.0$ and incubated over night with 96-well plates pre-treated for 30 min with methyl-/vinyletheranhydride-copolymer in DMSO, followed by $2.5 \mathrm{~h}$ with adipic acid dihydrazide. The resulting Schiff's bases are reduced to stable alkylamine bonds covalently linking the GAGs to the dish by incubating them for 30 min with $1 \%$ sodium 
cyanoborohydride in methanol. The prepared surfaces were then washed with $10 \mathrm{mM}$ Tris/HCl, $50 \mathrm{mM}$ sodium chloride, pH 7.4 (TBS) and blocked for $2 \mathrm{~h}$ with $2 \%$ BSA in TBS. For non-specific binding untreated wells were incubated with $2 \%$ BSA in TBS and untreated wells with no additions were used as positive control for growth factor binding. 0 - $100 \mathrm{ng} / \mathrm{ml}(0-5 \mathrm{ng})$ of rhBMP-4 in phosphate buffered saline (PBS) with the addition of $1 \%$ BSA were then incubated with the prepared surfaces over night at $4^{\circ} \mathrm{C}$.

Enzyme linked immunosorbent assay (ELISA). The amount of bound growth factors was determined directly on the MaxiSorp ${ }^{\mathrm{TM}}$ 96-well microtiter plates coated with GAGs (Direct-ELISA) or indirectly in the solution containing non-bound rhBMP-4 (Sandwich-ELISA) recovered from the supernatant after incubation with immobilized GAGs. A calibration curve ranging from $0 \mathrm{ng} / \mathrm{ml}-50$ $\mathrm{ng} / \mathrm{ml}(0-2.5 \mathrm{ng})$ in $1 \% \mathrm{BSA} / \mathrm{PBS}$ was prepared for rhBMP-4 to calculate the amount of non-bound growth factors. All binding data are the average of triplicates and statistically analyzed by two-way ANOVA with means comparison using Tukey test.

In brief, up to $5 \mathrm{ng}$ of rhBMP-4 were incubated for $16 \mathrm{~h}$ with immobilized GAGs on 96-well ELISAplates at $4^{\circ} \mathrm{C}$ for binding to reach equilibrium. The supernatants were removed and transferred to another MaxiSorp ${ }^{\mathrm{TM}}$ 96-well microtiter plate pre-coated with growth factor specific capture antibodies for Sandwich- ELISA and blocked with 1\% (w/v) BSA, 5\% Sucrose (w/v), 0.05\% (v/v) Tween 20 in PBS.

The Direct-ELISA plates were blocked with 3\% (w/v) BSA, 0.05\% (v/v) Tween 20 in PBS for $1.5 \mathrm{~h}$ at room temperature, washed with $0.05 \%(\mathrm{v} / \mathrm{v})$ Tween 20 in PBS, and then incubated with primary antibody for $2 \mathrm{~h}$ at room temperature. The plates were incubated with biotin-conjugated goat anti-mouse antibodies for $1 \mathrm{~h}$ followed by alkaline phosphatase-conjugated streptavidin for $1 \mathrm{~h}$. Antibodies and alkaline phosphatase-conjugated streptavidin were diluted in $0.5 \%(\mathrm{w} / \mathrm{v}) \mathrm{BSA}, 0.05 \%(\mathrm{v} / \mathrm{v})$ Tween 20 in PBS, respectively. Inbetween each incubation step the wells were washed with $0.05 \%$ Tween in PBS. The wells were developed with 4-nitrophenylphosphate disodium salt in $0.1 \mathrm{M}$ glycine, $1 \mathrm{mM} \mathrm{MgCl}_{2}, 1$ 
$\mathrm{mM} \mathrm{ZnCl} 2, \mathrm{pH} 10.4$. The reaction was allowed to proceed for $10-20 \mathrm{~min}$ and then stopped with $3 \mathrm{M}$ $\mathrm{NaOH}$ and measured at $\mathrm{A}_{405 \mathrm{~nm}}$ using a microtiter plate reader.

After washing with $0.05 \%(\mathrm{v} / \mathrm{v})$ Tween 20 in PBS the Sandwich-ELISA plates were incubated with biotinylated growth factor specific antibodies diluted in PBS for $2 \mathrm{~h}$. Following a washing step streptavidin-horseradish peroxidase diluted in 1\% BSA/PBS was added to the wells for 20 min. The plates were developed with 3,3',5,5'-tetramethylbenzidine liquid substrate. The reaction was stopped with $1 \mathrm{M} \mathrm{H}_{2} \mathrm{SO}_{4}$ and measured at $\mathrm{A}_{450 \mathrm{~nm}}$ using a microtiter plate reader.

Competitive binding inhibition using dissolved GAGs. A competitive inhibition ELISA assay using dissolved GAGs to compete for growth factor binding to a sHya2.8-immobilized surface was further employed to assess the differences in affinity and the specificity of the interaction between GAGs and rhBMP-4.

For competition experiments, the procedure was as detailed as for Direct-ELISA, except that a constant concentration of $2.5 \mathrm{ng}$ of growth factor was incubated with variable concentrations $\left(10^{-2}-10^{-9}\right.$ $\mathrm{g} / \mathrm{ml}$ ) of dissolved competitors in $1 \%(\mathrm{w} / \mathrm{v})$ BSA, PBS. The data were fitted with a four-parameter logistic equation and plotted on a semi-logarithmic scale using non-linear regression with Origin software. Data were plotted for each protein, and in the following the amount of GAG required to inhibit $50 \%$ of binding to each surface was determined. All binding data are the average of triplicates.

\section{Results}

Characterization of sHya and CS. The synthesis of sHya derivatives using different $\mathrm{SO}_{3}$ complexes $\left(\mathrm{SO}_{3}\right.$-pyridine and $\mathrm{SO}_{3}$-DMF) as sulfating agents led to a low-sulfated sHya (sHya1.0, D.S. = 1.0) and a high-sulfated form (sHya2.8, D.S. = 2.8; Figure 1). 


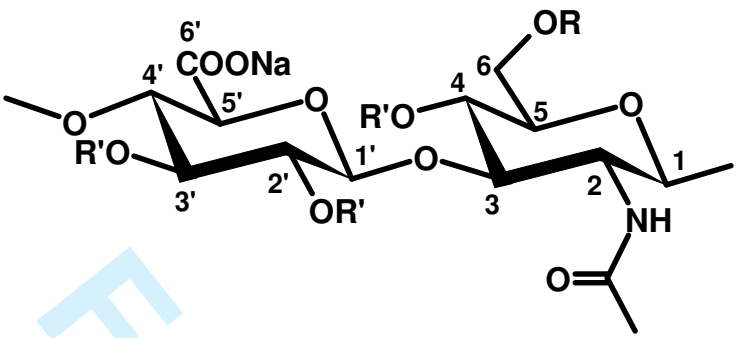

hyaluronic acid, Na-salt (Hya): R, R' = H low-sulfated Hya (sHya1.0): $\mathrm{R}=\mathrm{SO}_{3} \mathrm{Na}, \mathrm{R}^{\prime}=\mathrm{H}$ high-sulfated Hya (sHya2.8): $\mathrm{R}=\mathrm{SO}_{3} \mathrm{Na}, \mathrm{R}^{\prime}=\mathrm{H}$ or $\mathrm{SO}_{3} \mathrm{Na}$
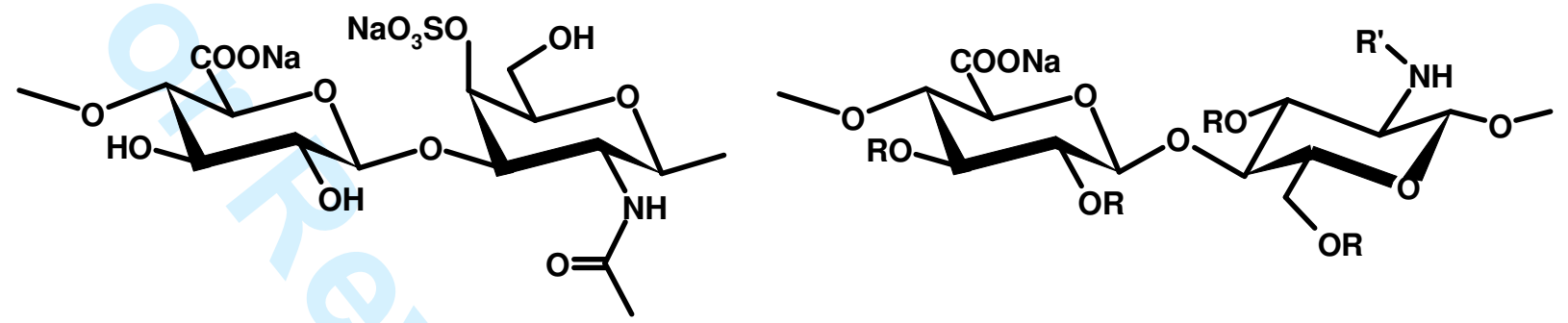

chondroitin-4-sulfate, Na-salt (CS-A)

heparan sulfate $(\mathrm{HA}): \mathrm{R}=\mathrm{H}$ or $\mathrm{SO}_{3} \mathrm{Na} ; \mathrm{R}^{\prime}=\mathrm{COCH}_{3}$ or $\mathrm{SO}_{3} \mathrm{Na}$

Figure 1. Chemical structures of glycosaminoglycans tested for binding to rhBMP-4.

${ }^{13}$ C-NMR spectroscopy was employed to study the distribution of introduced sulfate groups within the disaccharide repeating unit of Hya. Comparing the ${ }^{13} \mathrm{C}-\mathrm{NMR}$ spectra of unsubstituted Hya and both the sulfated derivatives sHya1.0 and sHya2.8 (Figure 2), a low-field shift of the C-6-signal of the primary $\mathrm{CH}_{2}$-group in the $\mathrm{N}$-actetyl-glucosamine unit from $61 \mathrm{ppm}$ to $68 \mathrm{ppm}$ was observed after sulfation. A nearly complete sulfation of the C-6-position might therefore be assumed even in the case of the low-sulfated derivative sHya1.0.

Except for C-6, the carbon signals of the low-sulfated sHya1.0 show chemical shifts similar to the ones of unsubstituted Hya allowing a full assignment of the different carbon signals. In contrast the complete assignment of the signals in the ${ }^{13} \mathrm{C}-\mathrm{NMR}$ spectra of the high-sulfated sHya2.8 is difficult because of the complexity of the obtained spectrum. 
Figure 2. ${ }^{13} \mathrm{C}$-NMR spectra for substituent distribution of unmodified and of modified Hya sHya2.8 and sHya1.0.

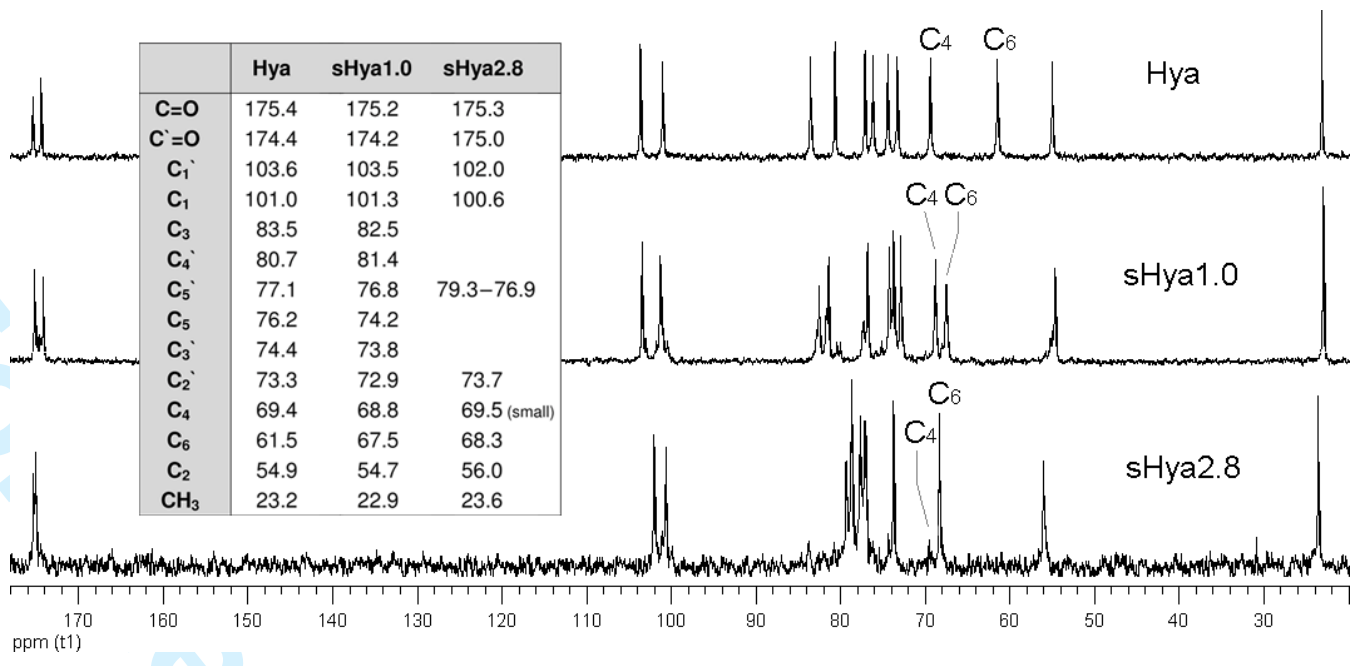

Nevertheless, from the obtained spectroscopic data it can be postulated that in the high-sulfated derivative sHya2.8 besides a complete sulfation of the primary OH-group at C-6, sulfation also occurs, to a lesser extent on the remaining secondary $\mathrm{OH}$ groups of the $\mathrm{N}$-acetylglucosamine unit $(\mathrm{C}-4)$ and the glucuronic acid unit (C-2', C-3'). The C-4-position might be sulfated to a slightly higher extent than both of the OH-groups at C-2' and C-3'. As shown in Table 1, the Hya educts undergo a decrease in their molecular weight during the sulfation reaction. Starting from Hya with a weight-average molecular weight $\left(\mathrm{M}_{\mathrm{W}}\right)$ of $10 \mathrm{E}+6 \mathrm{~g} \mathrm{~mol}^{-1}$, the $\mathrm{M}_{\mathrm{w}}$ of sHya dropped down to less than $10 \mathrm{E}+5 \mathrm{~g} \mathrm{~mol}^{-1}$. Interestingly, sulfation leads to a molecular weight distribution which is considerably narrower as is illustrated by the determined lower PD values. As LLS detection can lead to a discrimination of low molecular-weight fractions of the measured polymers $(\sim 5-50,000 \mathrm{~g} / \mathrm{mol})$ resulting in inaccurately narrow molecular weight distributions, the calculation of polydispersity PD was performed on the basis of $M_{n}$ and $M_{w}$ values measured by RI detection.

\begin{tabular}{llllll}
\hline Sample & Hya & sHya2.8 & sHya1.0 & CS & HS \\
\hline Sulfur content [\%] & - & 13.1 & 6.6 & 4.9 & 4.7 \\
\hline D.S. & 0.0 & 2.8 & 1.0 & 0.9 & n.d.
\end{tabular}




$\begin{array}{llllll}\text { Mn }\left[\mathrm{g} \mathrm{mol}^{-1}\right] & 1,020,000(\mathrm{LLS}) & 46,000(\mathrm{LLS}) & 29,000(\mathrm{LLS}) & 15,000(\mathrm{LLS}) & \text { n.d. } \\ & 395,000(\mathrm{RI}) & 72,000(\mathrm{RI}) & 38,000(\mathrm{RI}) & 49,000(\mathrm{RI}) & \\ \mathrm{Mw}\left[\mathrm{g} \mathrm{mol}^{-1}\right] & 1,175,000(\mathrm{LLS}) & 74,000(\mathrm{LLS}) & 33,000(\mathrm{LLS}) & 18,000(\mathrm{LLS}) & \text { n.d. } \\ & 1,895,000(\mathrm{RI}) & 121,000(\mathrm{RI}) & 79,000(\mathrm{RI}) & 67,000(\mathrm{RI}) & \\ \text { PD } & 4.80 & 1.68 & 2.08 & 1.37 & \text { n.d. }\end{array}$

TABLE 1: Characteristics of Hya, synthesized Hya derivatives and CS. D.S., number-average (Mn) and weight-average (Mw) molecular weights as determined by LLS detection and RI detection. Molecular weight distributions (polydispersity index: PD) based on the values calculated from RI detection; n.d.: not determined.

SPR-analysis of GAG-growth factor interaction. Varying concentrations of GAGs were injected over the sensor chip surface with immobilized rhBMP-4, and the change in response was evaluated as a function of time. Initially, HBS-EP running buffer was injected over the surface, and the response was taken as the reference value. In the following the GAG solution was injected (Figure 3, Arrow A), and the increasing response corresponded with the association of the GAGs with rhBMP-4. In the final phase, the fluid passing over the surface again contained only running buffer (Figure 3, Arrow D), which allowed the carbohydrate to dissociate from the protein due to a concentration gradient, thereby causing a slight decrease in the measured response. Resulting data were corrected by the response of a blank flow cell treated only with coupling chemistry. Figure 3 depicts the sensorgrams for the binding of a sHya2.8 concentration series to rhBMP-4, displaying an increasing response with growing concentration. 


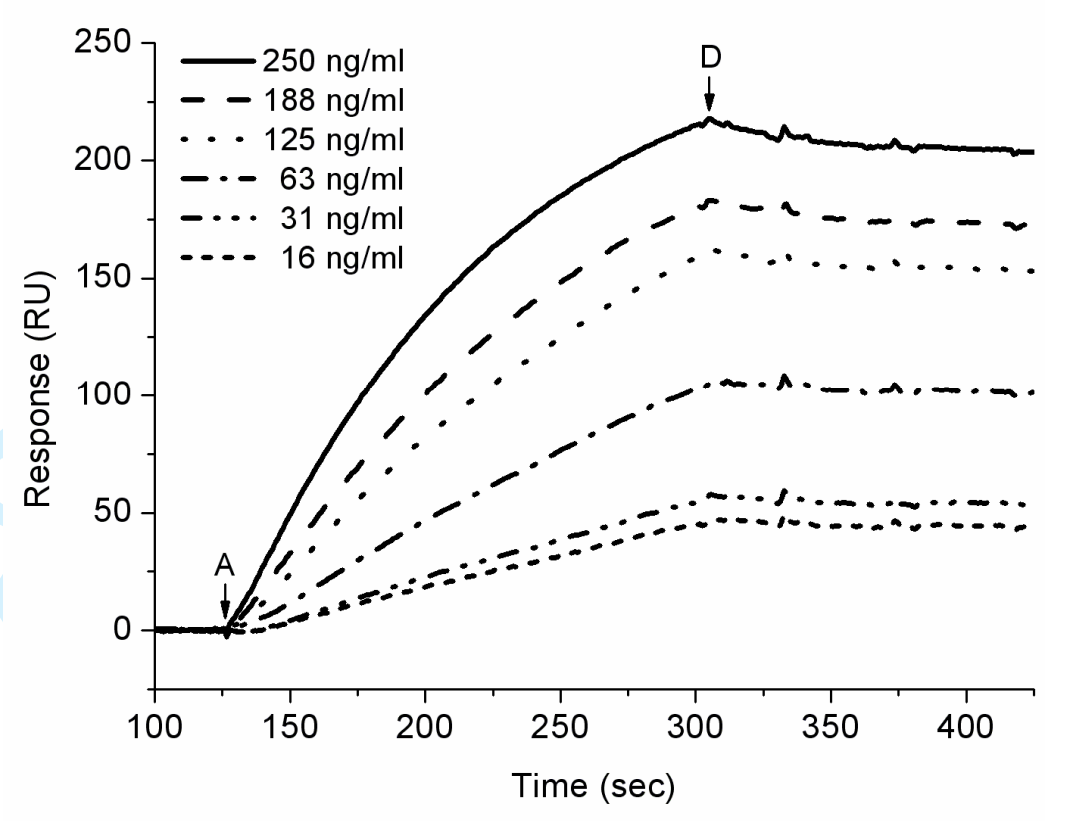

Figure 3. SPR sensorgrams of the interaction of sHya 2.8 with immobilized rhBMP-4 at $\mathrm{T}=25^{\circ} \mathrm{C}$. Sensorgrams of the rhBMP-4/sHya2.8 interaction were run at different concentrations of sHya2.8 (in $\mathrm{ng} / \mathrm{ml}$ ) in running buffer: $16,31,63,125,188,250$. The flow rate was $30 \mu 1 / \mathrm{min}$. Arrow A represents the starting point of the sample injection or the beginning of the association phase. Arrow D represents the end of the sample injection or the beginning of the dissociation phase.

Figure 4 displays a comparison of the interactions of different GAGs with rhBMP-4 at certain concentrations. The response for the interaction of unmodified Hya, CS, and HS at $50 \mu \mathrm{g} / \mathrm{ml}$ is considerably smaller than for sHya1.0 at $10 \mu \mathrm{g} / \mathrm{ml}$. To reach an analogous response to the interaction between rhBMP-4 and sHya2.8, sHya1.0 concentrations in the range of $\mu \mathrm{g} / \mathrm{ml}$ are necessary compared to $\mathrm{ng} / \mathrm{ml}$ for sHya2.8 (Figure 3 and 4 ).

The affinity of GAGs for rhBMP-4 was quantified by determining the overall dissociation constant $\mathrm{K}_{\mathrm{D}}\left(\mathrm{K}_{\mathrm{D}}=\mathrm{k}_{\mathrm{off}} / \mathrm{k}_{\mathrm{on}}\right)$, where $\mathrm{k}_{\mathrm{off}}$ and $\mathrm{k}_{\mathrm{on}}$ represent dissociation and association rate constants calculated from the dissociation and the association phase of the curves, respectively (Table 2). 


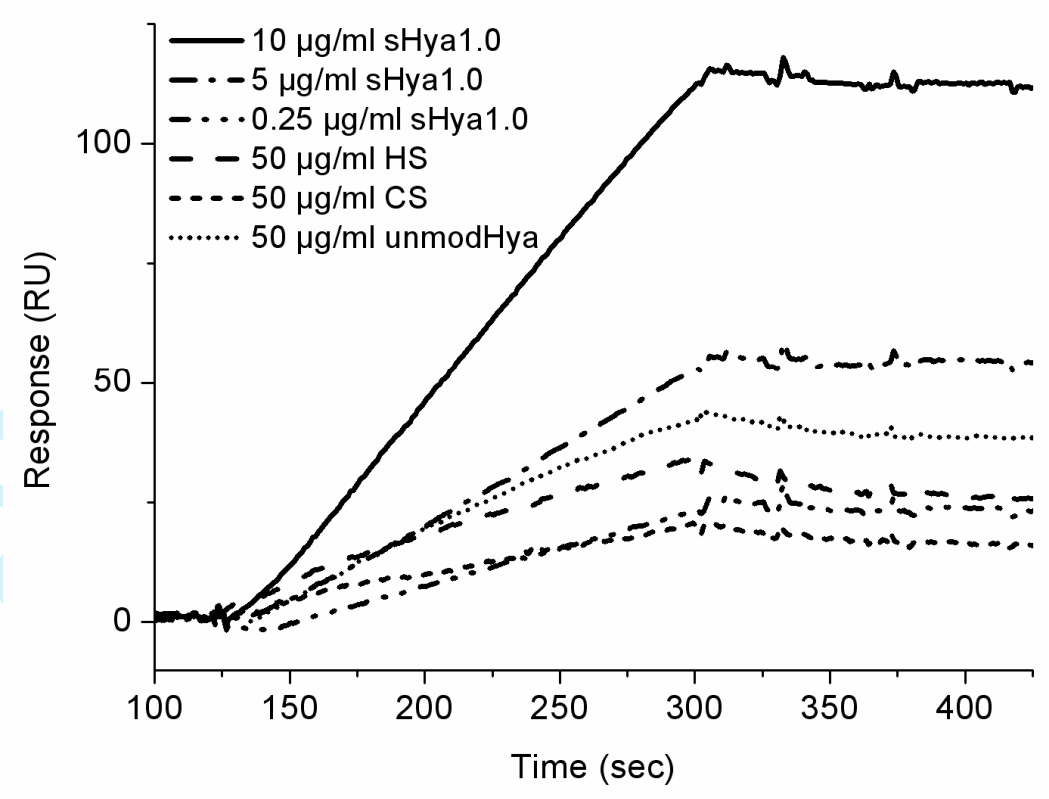

Figure 4. SPR sensorgrams of the interactions of different GAGs with immobilized rhBMP-4 at $\mathrm{T}=$ $25^{\circ} \mathrm{C}$. Sensorgrams for sHya1.0 were run at concentrations of $250 \mathrm{ng} / \mathrm{ml}, 5 \mu \mathrm{g} / \mathrm{ml}$ and $0.25 \mathrm{mg} / \mathrm{ml}$, those for unmodified Hya, CS, and HS at $50 \mu \mathrm{g} / \mathrm{ml}$. The flow rate was $30 \mu 1 / \mathrm{min}$.

\begin{tabular}{lllll}
\hline GAG & $\mathrm{k}_{\mathrm{on}}[1 / \mathrm{Ms}]$ & $\mathrm{k}_{\mathrm{off}}[1 / \mathrm{s}]$ & $\mathrm{K}_{\mathrm{D}}[\mathrm{nM}]$ & $\mathrm{Chi}^{2}$ \\
derivative & & & & \\
\hline sHya2.8 & $2.26 * 10 \mathrm{E} 7$ & $4.58 * 10 \mathrm{E}-4$ & 0.020 & 7.78 \\
& $2.25 * 10 \mathrm{E} 7$ & $2.94 * 10 \mathrm{E}-4$ & 0.013 & 7,04 \\
sHya1.0 & $3.91 * 10 \mathrm{E} 4$ & $4.47 * 10 \mathrm{E}-4$ & 11.4 & 12.5 \\
& $4.42 * 10 \mathrm{E} 4$ & $3.24 * 10 \mathrm{E}-4$ & 7.3 & 7.78 \\
HS & $2.73 * 10 \mathrm{E} 2$ & $1.49 * 10 \mathrm{E}-3$ & 5450 & 1.84 \\
& $2.68 * 10 \mathrm{E} 2$ & $1.32 * 10 \mathrm{E}-3$ & 4940 & 0.62 \\
\hline
\end{tabular}

TABLE 2: Kinetic rate constants $\left(k_{\text {on }}\right.$ and $k_{\text {off }}$ ) and equilibrium binding constants $\left(K_{D}\right)$ for the interaction of GAGs and immobilized rhBMP-4 after fitting with BIAevaluation software 4.1 applying a $A+B=A B$ Langmuir binding model. 
The differences of the association rates between the sulfated GAGs were evaluated after fitting the binding data to an $\mathrm{A}+\mathrm{B}=\mathrm{AB}$ Langmuir model with BIAevaluation software 4.1 (Table 2). Naturally sulfated HS (2.68 - 2.73*10E+2 1/Ms) exhibits a five orders of magnitude lower $\mathrm{k}_{\text {on }}$ value compared to sHya2.8 (2.25 - 2.26*10E+7 1/Ms), and a two orders of magnitude lower $\mathrm{k}_{\text {on }}$ value than sHya1.0 (3.91 4.42*10E+4 1/Ms). While sHya2.8 and sHya1.0 had comparable dissociation rate constants $\mathrm{k}_{\mathrm{off}}(2.94$ $4.58 * 10 \mathrm{E}-41 / \mathrm{s})$, they were three to five times lower than for HS $(1.32-1.49 * 10 \mathrm{E}-31 / \mathrm{s})$. The highest affinity, expressed as equilibrium dissociation constant $\mathrm{K}_{\mathrm{D}}$, could be determined for the interaction of rhBMP-4 with sHya2.8 (13 - 20 pM) followed by sHya1.0 (7.3 nM - $11.4 \mathrm{nM})$. HS exhibited a considerably lower affinity $(4.94 \mu \mathrm{M}-5.45 \mu \mathrm{M})$ to rhBMP-4 than sHya2.8 and sHya1.0.

Enzyme linked immunosorbent assay (ELISA). The binding of dissolved rhBMP-4 to GAGs immobilized on microtiter wells was performed by incubating up to $5 \mathrm{ng}$ of growth factor in $1 \%$ BSA/PBS. In the following Direct-ELISA the highest amount of bound rhBMP-4 was found in wells coated with the high-sulfated sHya2.8, closely followed by the low-sulfated form sHya1.0 $(\mathrm{p}<0.01)$ as shown in Figure 5. In contrast, for unmodified Hya, HS and CS coated surfaces significantly $(\mathrm{p}<0.001)$ less bound protein could be detected. However, all surfaces with immobilized GAGs retained significantly $(\mathrm{p}<0.001)$ more rhBMP-4 than those covered with BSA. 


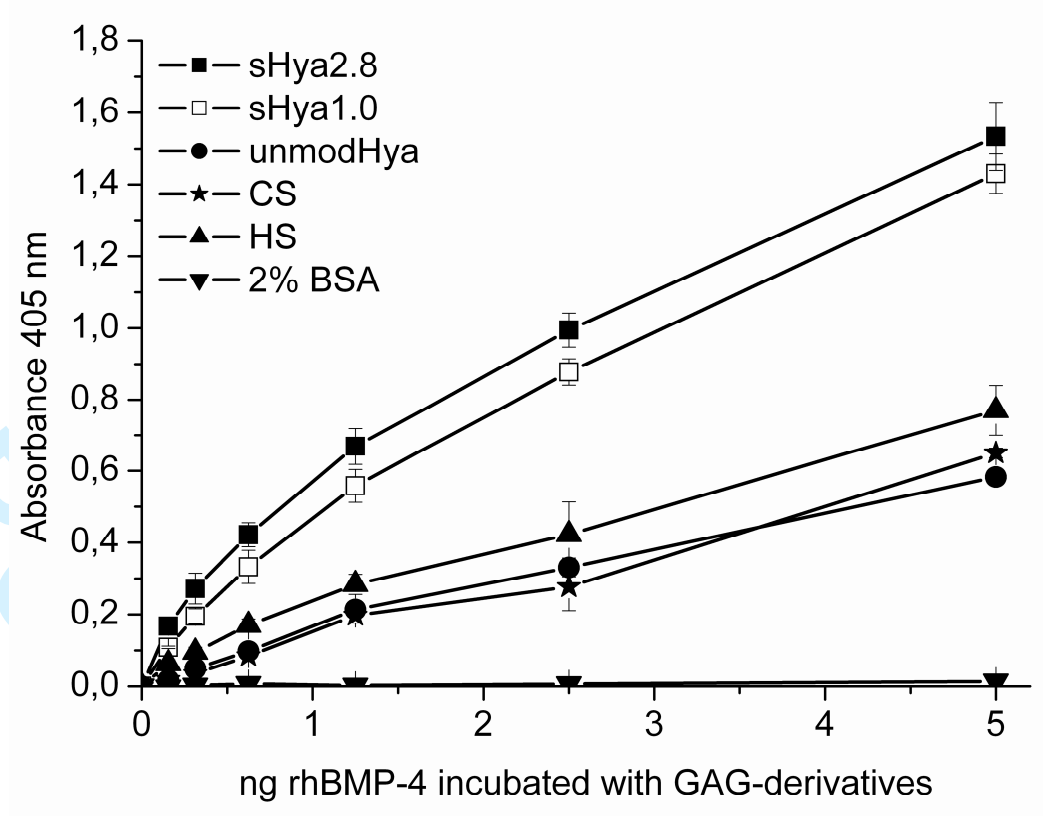

Figure 5. Direct-ELISA of up to $5 \mathrm{ng}$ rhBMP-4 in $1 \% \mathrm{BSA} / \mathrm{PBS}$ with GAGs immobilized on wells of MaxiSorp $^{\mathrm{TM}}$ 96-well microtiter plates. Surfaces coated with $2 \%$ BSA or untreated wells, the latter incubated with rhBMP-4 in PBS, served as negative or positive controls. The colorimetric reaction was followed at $\mathrm{A}_{405 \mathrm{~nm}}$. Binding data are the average of triplicates.

In the Sandwich-ELISA (Figure 6) the amount of the non-bound rhBMP-4 was determined in the supernatant as opposed to the bound growth factor detected directly on the surface of the Direct-ELISA plate. In the positive control no rhBMP-4 was found in the supernatant, demonstrating that everything had bound to the uncoated wells. In contrast, $\sim 1.8 \mathrm{ng}$ of rhBMP-4 (of $5 \mathrm{ng}$ originally incubated growth factor) were bound non-specifically to the surface in wells blocked with $2 \%$ BSA. Again coatings with GAGs retained significantly more rhBMP-4 than the BSA reference surface $(\mathrm{p}<0.001)$, with CS $(\sim 2.3$ ng of $5 \mathrm{ng}$ ) exhibiting the smallest amount bound and sHya2.8 the highest ( $3.5 \mathrm{ng}$ of $5 \mathrm{ng})$. As in the Direct-ELISA, sHya2.8 and sHya1.0 showed significantly more retained rhBMP-4 than unmodified Hya, HS and CS ( $\mathrm{p}<0.001$ and $\mathrm{p}<0.05$, respectively). Differences between HS and CS were not statistically significant. 


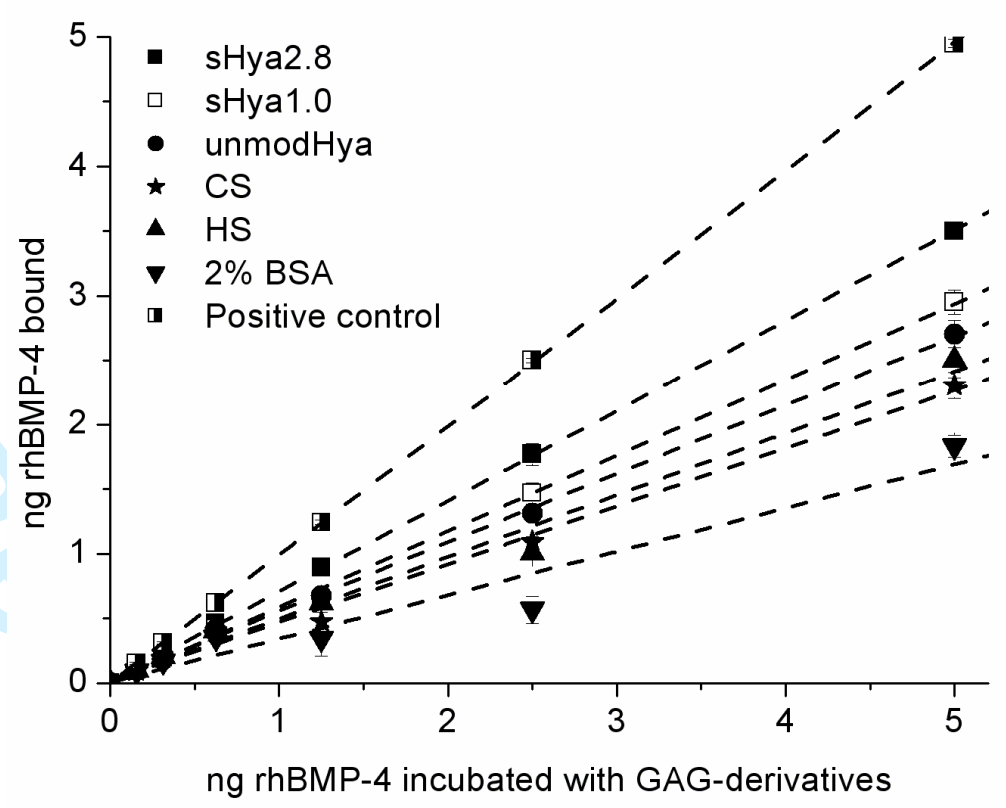

Figure 6. Sandwich-ELISA of the supernatants containing the non-bound rhBMP-4 recovered after incubation of up to $5 \mathrm{ng}$ rhBMP-4 with immobilized GAGs. The amount of bound growth factor was determined indirectly by comparison to a calibration curve of rhBMP-4. The colorimetric measurement was performed at $\mathrm{A}_{450 \mathrm{~nm}}$. Binding data are the average of triplicates.

Competitive inhibition of binding using dissolved GAGs. To confirm the specificity of the interactions of rhBMP-4 with GAGs, the abilities of different dissolved GAGs to compete with the interaction were investigated. A constant amount of rhBMP-4 was incubated with a surface displaying immobilized sHya2.8, but in the presence of varying amounts of free GAGs. The residual binding was determined at each concentration of dissolved GAGs by Direct-ELISA. Figure 7 shows that all dissolved GAGs tested are less potent competitors of the interaction of rhBMP-4 with immobilized sHya2.8 than dissolved sHya2.8 itself. The low-sulfated sHya1.0 (IC50 4.5*10E-7 g/ml $\pm 2.1 * 10 \mathrm{E}-7$ $\mathrm{g} / \mathrm{ml}$ ) exhibited two orders of magnitude weaker competition abilities than sHya2.8 (IC50 1.3*10E-9 $\mathrm{g} / \mathrm{ml} \pm 6.8 * 10 \mathrm{E}-9 \mathrm{~g} / \mathrm{ml})$. In comparison to the sulfated Hya derivatives, unmodified Hya, CS and HS are much weaker competitors. The results obtained showed a ranking of binding affinities as sHya2.8 > sHya1.0 > unmodified Hya $\approx \mathrm{CS}>\mathrm{HS}$. 


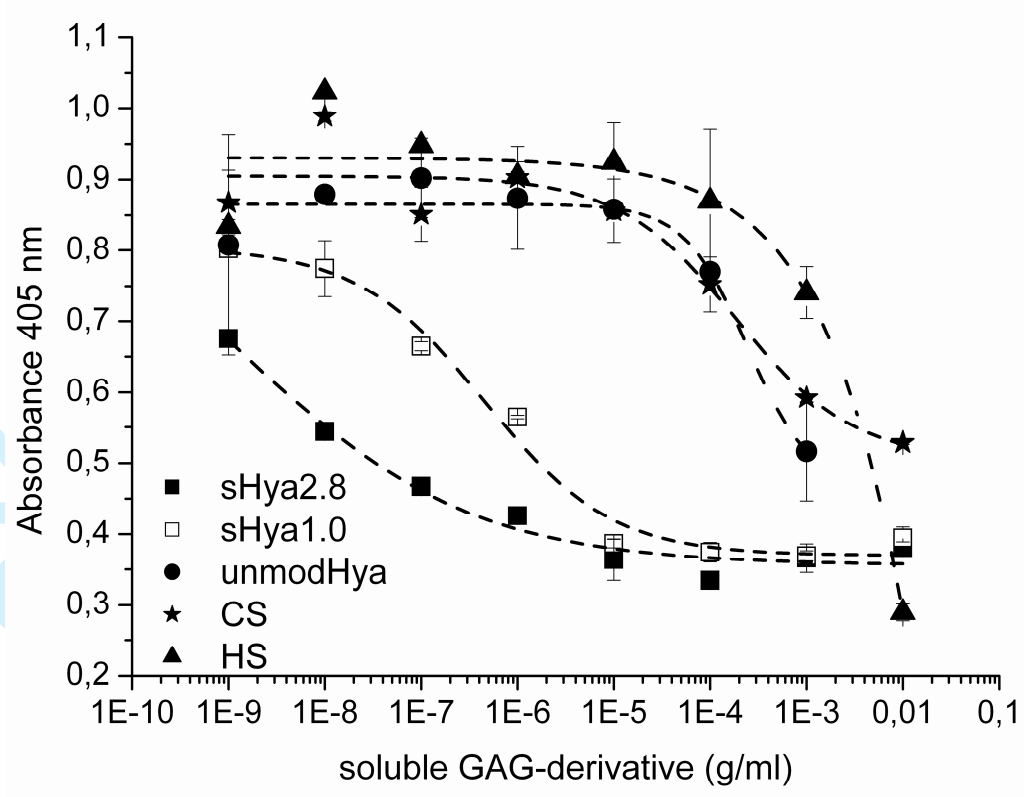

Figure 7. Competitive inhibition of rhBMP-4 binding to immobilized sHya2.8 using varying concentrations of dissolved GAGs. A Direct-ELISA of $2.5 \mathrm{ng}$ rhBMP-4 in 1\% BSA/PBS was performed and the colorimetric measurement was recorded at $\mathrm{A}_{405 \mathrm{~nm}}$. Values are the means of triplicates and data are representative of two separate experiments.

\section{Discussion}

The major goal of this study was to investigate the growth factor binding abilities of Hya modified with sulfate groups, aiming at a deeper understanding of structure-function relationships of Hya derivative interactions with biological mediators.

To this end high-sulfated Hya (D.S. 2.8) and low-sulfated Hya (D.S. 1.0) were prepared and characterized. The ${ }^{13} \mathrm{C}-\mathrm{NMR}$ spectra of sHya proved that the primary OH-group at the C-6 position in $\mathrm{N}$-acetylglucosamine is preferentially sulfated, followed by the secondary OH-group at C-4. This sulfation of the C-4 and the C-6 seems to be of special importance for binding to BMP-4, as Miyazaki et al. could show that C-4, C-6-disulfation of galactosamine in chondroitin sulfate E is necessary for high affinity $^{30}$, while BMP-7, another member of the TGF- $\beta$ growth factor superfamily, requires 6-O- and N- 
sulfation of the $\mathrm{N}$-acetyl-glucosamine unit for binding to $\mathrm{HS}^{31}$. The $\mathrm{C}-6$ modification is the only sulfate group that is post-synthetically edited by the cell surface sulfatases, and in addition to influencing binding has been shown to affect growth factors signaling, specifically for Wnt, FGF2 and BMP ${ }^{20}$.

Several studies have quantified the rate constants as well as the affinities of interactions between proteins and GAGs, in particular heparin or HS chains using optical biosensors ${ }^{24,29,32-34}$, clearly demonstrating the ability of the SPR technique to generate kinetic data as well as binding constants in this context. Our own data proved a concentration dependency of the interaction between GAGs and rhBMP-4 (Figure 3). The results indicated that the sulfated Hya derivatives exhibit fast on-rate $\left(\mathrm{k}_{\mathrm{on}}\right)$ and slow off-rate $\left(\mathrm{k}_{\mathrm{off}}\right)$ kinetics for rhBMP-4 with $\mathrm{K}_{\mathrm{D}}$ values ranging from $13 \mathrm{pM}$ to $11.4 \mathrm{nM}$ (Table 2).

According to these $\mathrm{K}_{\mathrm{D}}$ values, sHya2.8 $(13-20 \mathrm{pM})$ exhibited the tightest binding to immobilized rhBMP-4, followed by sHya1.0 (7.3 - $11.4 \mathrm{nM})$ with the interaction being three orders of magnitude stronger for sHya2.8. This difference is due to the three orders of magnitude faster association rate of sHya2.8 (2.25 - 2.26*10E+7) compared to sHya1.0 (3.91 - 4.42*10E+4) Interestingly, HS with an only slightly lower sulfur content than sHya1.0 exhibited a three orders of magnitude lower affinity $\left(\mathrm{K}_{\mathrm{D}} 4.94\right.$ $-5.45 \mu \mathrm{M})$. This was due to its slow association rate of $2.68-2.73 * 10 \mathrm{E}+2$, as the the dissociation rate is only three to five times faster than for sHya2.8 and sHya1.0. The affinity constants of unmodified Hya and CS were not determined but assumed to be in a range comparable to HS, because the response for the interaction of $50 \mu \mathrm{g} / \mathrm{ml}$ GAGs with rhBMP-4 was comparable to HS (Figure 4). These results comply with those of Ruppert et al., who demonstrated the physical interaction between BMP-2 and immobilized heparin with a dissociation constant $\mathrm{K}_{\mathrm{D}}$ of approximately $20 \mathrm{nM}$ and a rapid association phase $\left(\mathrm{k}_{\mathrm{on}} \text {, of } 5.1+/-0.8 * 10 \mathrm{E}+5 \mathrm{M}^{-1} \mathrm{~s}^{-1}\right)^{29}$.

Obviously, besides the overall sulfate content the distribution of sulfate groups and therefore the number of high affinity binding sequences plays an important role in determining the affinity. Powell et al could show that chemically over-sulfated heparin was a marginally less effective competitor than heparin for binding to the FGF receptors FGFR1-Fc and FGFR2-Fc, indicating that the interactions are 
mediated by specific sequences within the heparin chains rather than by non-specific electrostatic interactions ${ }^{33}$.

In the presented study an $\mathrm{A}+\mathrm{B}=\mathrm{AB}$ Langmuir model provided the best fit for the interaction of GAGs and rhBMP-4. The selection of the model was based on the generated $\chi^{2}$ value (standard statistical measure of the closeness of the fit) being approximately in the same order of magnitude as the noise in the RU, and on the assumption that the GAG chains did not bridge multiple, adjacent proteins simultaneously if the lowest viable rhBMP-4 concentration was used. This was supported by biosensor interaction studies of immobilized heparin and dissolved BMP-2 that could also be confidently fitted on the basis of an $\mathrm{A}+\mathrm{B}=\mathrm{AB}$ model $^{29}$. Other reports suggested that some interactions between proteins and heparin or HS chains could not be characterized by a single-site binding model and hence were not kinetically homogeneous 35,36 . Obviously, the ability to characterize the interaction using single-site binding models is protein dependent ${ }^{37}$.

Although a number of other SPR-analyses used the same design of dissolved GAGs and immobilized proteins $^{32,34}$ to determine overall dissociation constants $K_{D}$, also showing high-sulfated GAGs to be better binders than low-sulfated ones, there are still some aspects to consider. Powell et al. for instance claimed that using GAGs as the dissolved binding partner could be problematic because of their low refractive indices, resulting in a small signal on mass basis ${ }^{37}$. Furthermore, the authors claim than the refractive index of GAGs could vary with the degree of sulfation, complicating a quantitative analysis for different chains. Consequently, Powell et al. suggest immobilizing GAGs on the sensor surface ${ }^{37}$. The commonly used technique here is biotin labelling of GAGs in combination with a streptavidinmodified sensor chip surface. However, this is also not without problems as the method of immobilization could affect the protein-GAG interactions. Osmond et al. demonstrated that heparin immobilization via the reducing terminus resulted in a higher binding capacity and in some cases a higher affinity for heparin-binding proteins compared to immobilization through intrachain uronic acids or amines ${ }^{38}$. Therefore directing biotinylation to the reducing end as developed for heparin and heparin- 
derived oligosaccharides is likely to be the least intrusive and most closely resembles a proteoglycan form of $\mathrm{GAGs}^{38-41}$.

In the ELISA studies performed using immobilized GAGs to support the findings of the SPR-analysis these were consequently attached to the polystyrene surface via their reducing ends ${ }^{42}$.

In general the ELISA results corroborated the ranking of binding to rhBMP-4 as determined in SPR analysis (Figure 4 and Table 2). The tightest binding could be demonstrated for sHya2.8, followed by sHya1.0, while unmodified Hya, CS and HS exhibited significantly weaker interactions (Figure 5 and 6). The results provide support for the assumption that the nature of binding is not severely affected by which partner is immobilized and which is dissolved. To ensure that the observed effects where not due to different GAG concentrations immobilized on the plate, a competition ELISA in which dissolved GAGs compete for binding to rhBMP-4 with immobilized sHya2.8 was performed, which largely confirmed the SPR and ELISA results.

It revealed that dissolved sHya2.8 was the most potent competitor for the interaction of dissolved rhBMP-4 with immobilized sHya2.8 (IC50 1.3*10E-9 g/ml $\pm 6.8 * 10 \mathrm{E}-9 \mathrm{~g} / \mathrm{ml}$ ), while sHya.1.0 (IC50 $4.5 * 10 \mathrm{E}-3 \mathrm{~g} / \mathrm{ml} \pm 2.1 * 10 \mathrm{E}-7 \mathrm{~g} / \mathrm{ml}$ ) exhibited competition abilities which are two orders of magnitude weaker (Figure 7). The results obtained prove the specificity of the interactions of rhBMP-4 with GAGs. The differences of the IC50-values are also close to the differences in affinity $\left(\mathrm{K}_{\mathrm{D}}\right)$ between these Hya derivatives determined by SPR (Table 2). Since results from both ELISA strategies are consistent, we assume comparable immobilization levels of all GAGs on direct ELISA plates.

Interestingly in all three experimental approaches the unmodified, non-sulfated Hya seems to exhibit a binding strength comparable to CS and HS. This may be due in part to the fact that for the same mass/volume ratios the total number of disaccharide units is slightly higher for unmodified Hya than for CS and HS, as due to the missing sulfate groups the molecular weight of its disaccharide units is slightly lower. Because the interaction with rhBMP-4 is mainly electrostatic, additional non-sulfated but negatively charged disaccharide units might cause an increased binding response. We furthermore 
speculate that an unsulfated GAG such as Hya may have a different binding profile due to possible additional binding forces like van der Waals and hydrogen bonding ${ }^{43}$.

The obtained differences in binding affinities of the sulfated GAGs could be explained by the partial sulfation of the C-4 position in addition to a complete sulfation of the C-6 position in the $\mathrm{N}$ acetylglucosamine unit of sHya2.8, as Miyazaki et al. found that the C-4, C-6-disulfated structure of the $\mathrm{N}$-acetylglucosamine unit is important for strong binding to BMP- $4^{30}$. The strongest interaction was displayed by sHya2.8, which is fully sulfated in the C-6 and partially in the C-4 position. SHya1.0 and CS with their significantly lower binding, on the other hand, are primarily monosulfated. The fact that CS bound still less than sHyal.0 despite a comparable degree of total sulfation may be due to the fact that sHya1.0 is sulfated mainly in the C-6 position and marginally in C-4, while CS carries most sulfate groups in the $\mathrm{C}-4$ position. This hypothesis has to be confirmed with a greater range of structures, especially a sHya specifically sulfated in C-4 rather than C-6 position, to convincingly distinguish positional from density effects.

\section{Conclusion}

We have demonstrated specific interactions between sHya derivatives and rhBMP-4 with different binding affinities depending on sulfation degree, with an additional influence of the sulfate position. The differentially sulfated Hyas are therefore promising model substances for reaching a further understanding of the structure-function relationships of GAGs. Furthermore, they might be interesting candidates for bioengineered coatings to improve the biological properties of biomaterials by possibly attracting growth factors from the blood stream.

Acknowledgement. We thank Prof. Dr. Mario Mörl, Institute of Biochemistry, Universität Leipzig, Germany for kind permission to use the BIAcore 3000 instrument. We further acknowledge financial support by the DFG [PAK 105, TRR 67] and the BMBF [01EZ074]. 


\section{References}

(1) Bulpitt, P.; Aeschlimann, D. J. Biomed. Mater. Res., 1999, 47, (2), 152-169.

(2) Morra, M. Biomacromolecules, 2005, 6, (3), 1205-1223.

(3) Laurent, T. C.; Fraser, J. R. Faseb J., 1992, 6, (7), 2397-2404.

(4) Ishida, O.; Tanaka, Y.; Morimoto, I.; Takigawa, M.; Eto, S. J. Bone Miner. Res., 1997, 12, (10), 1657-1663.

(5) Lesley, J.; Hascall, V. C.; Tammi, M.; Hyman, R. J. Biol. Chem., 2000, 275, (35), 26967-26975.

(6) Allison, D. D.; Grande-Allen, K. J. Tissue Eng., 2006, 12, (8), 2131-2140.

(7) McKee, C. M.; Penno, M. B.; Cowman, M.; Burdick, M. D.; Strieter, R. M.; Bao, C.; Noble, P. W. J. Clin. Invest., 1996, 98, (10), 2403-2413.

(8) Evanko, S. P.; Angello, J. C.; Wight, T. N. Arterioscler. Thromb. Vasc. Biol., 1999, 19, (4), 1004-1013.

(9) King, S. R.; Hickerson, W. L.; Proctor, K. G. Surgery, 1991, 109, (1), 76-84.

(10) Knudson, C. B.; Knudson, W. Faseb J., 1993, 7, (13), 1233-1241.

(11) Huang, L.; Cheng, Y. Y.; Koo, P. L.; Lee, K. M.; Qin, L.; Cheng, J. C.; Kumta, S. M. J. Biomed. Mater. Res. A, 2003, 66, (4), 880-884.

(12) Prestwich, G. D.; Kuo, J. W. Curr. Pharm. Biotechnol., 2008, 9, (4), 242-245.

(13) Hong, L.; Tabata, Y.; Yamamoto, M.; Miyamoto, S.; Yamada, K.; Hashimoto, N.; Ikada, Y. J. Biomater. Sci. Polym. Ed., 1998, 9, (9), 1001-1014.

(14) Yamamoto, M.; Tabata, Y.; Ikada, Y. J. Biomater. Sci. Polym. Ed., 1998, 9, (5), 439-458.

(15) Kirker-Head, C. A. Adv. Drug Deliv. Rev., 2000, 43, (1), 65-92.

(16) Yamada, T.; Sawada, R.; Tsuchiya, T. Biomaterials, 2008, 29, (26), 3503-3513.

(17) Nagahata, M.; Tsuchiya, T.; Ishiguro, T.; Matsuda, N.; Nakatsuchi, Y.; Teramoto, A.; Hachimori, A.; Abe, K. Biochem. Biophys. Res. Commun., 2004, 315, (3), 603-611.

(18) Gallagher, J. T.; Turnbull, J. E.; Lyon, M. Int. J. Biochem., 1992, 24, (4), 553-560.

(19) Maccarana, M.; Sakura, Y.; Tawada, A.; Yoshida, K.; Lindahl, U. J. Biol. Chem., 1996, 271, (30), 17804-17810.

(20) Lamanna, W. C.; Kalus, I.; Padva, M.; Baldwin, R. J.; Merry, C. L.; Dierks, T. J. Biotechnol., 2007, 129, (2), 290-307.

(21) Bourin, M. C.; Lindahl, U. Biochem. J., 1993, 289 (Pt 2), 313-330.

(22) Cardin, A. D.; Weintraub, H. J. Arteriosclerosis, 1989, 9, (1), 21-32.

(23) Margalit, H.; Fischer, N.; Ben-Sasson, S. A. J. Biol. Chem., 1993, 268, (26), 19228-19231.

(24) Rahmoune, H.; Chen, H. L.; Gallagher, J. T.; Rudland, P. S.; Fernig, D. G. J. Biol. Chem., 1998, 273, (13), 7303-7310.

(25) Chen, Q.; Sivakumar, P.; Barley, C.; Peters, D. M.; Gomes, R. R.; Farach-Carson, M. C.; Dallas, S. L. J. Biol. Chem., 2007, 282, (36), 26418-26430.

(26) Kreuger, J.; Spillmann, D.; Li, J. P.; Lindahl, U. J. Cell. Biol., 2006, 174, (3), 323-327.

(27) Vlodavsky, I.; Folkman, J.; Sullivan, R.; Fridman, R.; Ishai-Michaeli, R.; Sasse, J.; Klagsbrun, M. Proc. Natl. Acad. Sci. U.S.A, 1987, 84, (8), 2292-2296.

(28) Takada, T.; Katagiri, T.; Ifuku, M.; Morimura, N.; Kobayashi, M.; Hasegawa, K.; Ogamo, A.; Kamijo, R. J. Biol. Chem., 2003, 278, (44), 43229-43235.

(29) Ruppert, R.; Hoffmann, E.; Sebald, W. Eur. J. Biochem., 1996, 237, (1), 295-302.

(30) Miyazaki, T.; Miyauchi, S.; Tawada, A.; Anada, T.; Matsuzaka, S.; Suzuki, O. J. Cell. Physiol., 2008, 217, (3), 769-777.

(31) Irie, A.; Habuchi, H.; Kimata, K.; Sanai, Y. Biochem. Biophys. Res. Commun., 2003, 308, (4), 858-865.

(32) Rathore, D.; McCutchan, T. F.; Garboczi, D. N.; Toida, T.; Hernaiz, M. J.; LeBrun, L. A.; Lang, S. C.; Linhardt, R. J. Biochemistry, 2001, 40, (38), 11518-11524.

(33) Powell, A. K.; Fernig, D. G.; Turnbull, J. E. J. Biol. Chem., 2002, 277, (32), 28554-28563. 
(34) Shen, B.; Shimmon, S.; Smith, M. M.; Ghosh, P. J. Pharm. Biomed. Anal., 2003, 31, (1), 83-93.

(35) Rux, A. H.; Lou, H.; Lambris, J. D.; Friedman, H. M.; Eisenberg, R. J.; Cohen, G. H. Virology, 2002, 294, (2), 324-332.

(36) Vives, R. R.; Sadir, R.; Imberty, A.; Rencurosi, A.; Lortat-Jacob, H. Biochemistry, 2002, 41, (50), 14779-14789.

(37) Powell, A. K.; Yates, E. A.; Fernig, D. G.; Turnbull, J. E. Glycobiology, 2004, 14, (4), 17 R-30R.

(38) Osmond, R. I.; Kett, W. C.; Skett, S. E.; Coombe, D. R. Anal. Biochem., 2002, 310, (2), 199-207.

(39) Delehedde, M.; Lyon, M.; Gallagher, J. T.; Rudland, P. S.; Fernig, D. G. Biochem. J., 2002, 366, (Pt 1), 235-244.

(40) Delehedde, M.; Lyon, M.; Vidyasagar, R.; McDonnell, T. J.; Fernig, D. G. J. Biol. Chem., 2002, 277, (14), 12456-12462.

(41) Duchesne, L.; Tissot, B.; Rudd, T. R.; Dell, A.; Fernig, D. G. J. Biol. Chem., 2006, 281, (37), 27178-27189.

(42) Satoh, A.; Kojima, K.; Koyama, T.; Ogawa, H.; Matsumoto, I. Anal. Biochem., 1998, 260, (1), 96-102.

(43) Day, A. J.; Prestwich, G. D. J. Biol. Chem., 2002, 277, (7), 4585-4588. 